\title{
Certain Properties of the Generalized Gauss Hypergeometric Functions
}

\author{
Praveen Agarwal* \\ Department of Mathematics, Anand International College of Engineering, Jaipur-303012, India
}

Received: 29 Aug. 2013, Revised: 26 Nov. 2013, Accepted: 27 Nov. 2013

Published online: 1 Sep. 2014

\begin{abstract}
In this paper, we aim at establishing certain integral transform and fractional integral formulas for the generalized Gauss hypergeometric functions, which was introduced and studied by Özergin et al. [J. Comput. Appl. Math. 235(2011), 4601-4610]. All the results derived here are of general character and can yield a number of (known and new) results in the theory of integral transforms and fractional integrals.
\end{abstract}

Keywords: Beta function; generalized beta functions; generalized Gauss hypergeometric functions; integral transforms; fractional integral operators.

\section{Introduction}

Recently, a function has attracted many researchers' attention due mainly to diverse applications, which are more general than the Beta type function $B(x, y)$, popularly known as generalized Beta type functions. These functions, as a part of the theory of confluent hypergeometric functions, are important special functions and their closely related ones are widely used in physics and engineering, therefore, they are of interest to physicists and engineers as well as mathematicians. Morover, generalized Beta functions [7,8] have played a pivotal role in the advancement of further research and have proved to be exemplery in nature.

Recently, Özergin et al. [14] introduced and studied some fundamental properties and characteristics of the generalized Beta type function $B_{p}^{(\alpha, \beta)}(x, y)$ in their paper and defined by (see, e.g., [14, p. 4602, Eq.(4)]; see also, $[13$, p.32, Chapter 4.]):

$B_{p}^{(\alpha, \beta)}(x, y)=\int_{0}^{1} t^{x-1}(1-t)^{y-1}{ }_{1} F_{1}\left(\alpha ; \beta ; \frac{-p}{t(1-t)}\right) d t$

$$
\begin{array}{r}
(\Re(p)>0(\Re(x), \Re(y), \Re(\alpha), \Re(\beta))>0 \\
\text { and } \left.B_{0}^{(\alpha, \beta)}(x, y)=B(x, y)\right),
\end{array}
$$

where $B(x, y)$ is a well known Euler's Beta function defined by:

$B(x, y)=\int_{0}^{1} t^{x-1}(1-t)^{y-1} d t(\Re(x)>0, \Re(y)>0)$.

Along with, generalized Beta function (1), Özergin et al. introduced and studied a family of the following potentially useful generalized Gauss hypergeometric functions defined as follows (see, e.g., [14, p. 4606, Section 3.]; see also, [13, p.39, Chapter 4.]):

$F_{p}^{(\alpha, \beta)}(a, b ; c ; z)=\sum_{n=0}^{\infty} a_{n} \frac{B_{p}^{(\alpha, \beta)}(b+n, c-b)}{B(b, c-b)} \frac{z^{n}}{n !}$

$$
(|z|<1) \text {, }
$$

where $\min (\mathfrak{R}(\alpha), \mathfrak{R}(\beta))>0 ; \Re(c)>\mathfrak{R}(b)>0$ and $\mathfrak{R}(p) \geq 0$.

Indeed, in their special case when $p=0$, the function $F_{p}^{(\alpha, \beta)}(a, b ; c ; z)$ would reduce immediately to the extensively-investigated Gauss hypergeometric function ${ }_{2} F_{1}($.$) . The { }_{2} F_{1}($.$) is the special case of the well known$ generalized hypergeometric series ${ }_{p} F_{q}($.$) defined by (see,$ e.g., [21, Section 1.5]; see also, [22]):

$$
\begin{array}{r}
{ }_{p} F_{q}\left[\begin{array}{l}
\alpha_{1}, \ldots, \alpha_{p} ; z \\
\beta_{1}, \ldots, \beta_{q} ;
\end{array}\right]=\sum_{n=0}^{\infty} \frac{\left(\alpha_{1}\right)_{n} \cdots\left(\alpha_{p}\right)_{n}}{\left(\beta_{1}\right)_{n} \cdots\left(\beta_{q}\right)_{n}} \frac{z^{n}}{n !} \\
={ }_{p} F_{q}\left(\alpha_{1}, \ldots, \alpha_{p} ; \beta_{1}, \ldots, \beta_{q} ; z\right),
\end{array}
$$

\footnotetext{
*Corresponding author e-mail: praveen.agarwal@anandice.ac.in, goyal.praveen2011@gmail.com
} 
where $(\lambda)_{n}$ is the Pochhammer symbol defined (for $\lambda \in \mathbb{C}$ ) by (see [21, p. 2 and pp. 4-6]):

$$
\begin{aligned}
& (\lambda)_{n}=\left\{\begin{array}{cc}
1 & (n=0) \\
\lambda(\lambda+1) \ldots(\lambda+n-1) & (n \in \mathbb{N})
\end{array}\right. \\
& =\frac{\Gamma(\lambda+n)}{\Gamma(\lambda)} \quad\left(\lambda \in \mathbb{C} \backslash \mathbb{Z}_{0}^{-}\right),
\end{aligned}
$$

and $\mathbb{Z}_{0}^{-}$denotes the set of nonpositive integers.

The above-mentioned detailed and systematic investigation by Özergin [13] (see also,[14]) was indeed motivated largely by the demonstrated potential for applications of the generalized Gauss hypergeometric function $F_{p}^{(\alpha, \beta)}$ and their special cases in many diverse areas of mathematical, physical, engineering and statistical sciences (see, for details, [13] and the references cited therein). Several further properties of the generalized Gauss hypergeometric functions and generating functions associated with them can be found in the subsequent developments presented in (see, for example, [13] and [6]). In the present sequel to these recent works, we propose to derive several integral transforms and image formulas for the generalized Gauss hypergeometric function $F_{p}^{(\alpha, \beta)}$ by applying a certain integral transforms (like, Beta transform, Laplace transform and Whittaker transforms) and the general pair of fractional integral operators involving Gauss hypergeometric function ${ }_{2} F_{1}$, respectively, which we introduce in Sections 2 and 3 respectively, below. We also consider some interesting special cases and consequences of our main results.

\section{Integral Transform of the Generalized Gauss Hypergeometric Functions}

In this section, we shall prove three theorems, which exhibit the connection between the integral transforms like Euler transform, Laplace transform and Wittaker transforms and the generalized Gauss hypergeometric type functions $F_{p}^{(\alpha, \beta)}(a, b ; c ; z)$ given by equation (3).

Theorem 1.The following Beta transform formula hold true:

$$
\begin{array}{r}
B\left\{F_{p}^{(\alpha, \beta)}(l+m, b ; c ; y z): l, m\right\} \\
=B(l, m) \sum_{n=0}^{\infty} l_{n} \frac{B_{p}^{(\alpha, \beta)}(b+n, c-b)}{B(b, c-b)} \frac{y^{n}}{n !} \\
=B(l, m) F_{p}^{(\alpha, \beta)}(l, b ; c ; y) \\
(\Re(p) \geq 0 ; l, m \in \mathbb{C} ;|y|<1),
\end{array}
$$

where the Beta transform of $f(z)$ is defined as [19]:

$B\{f(z): a, b\}=\int_{0}^{1} z^{a-1}(1-z)^{b-1} f(z) d z$.

Proof.On using the definition (7) and applying (3) to the Euler (Beta) transform of (6), we get

$$
\begin{array}{r}
\int_{0}^{1} z^{l-1}(1-z)^{m-1} F_{p}^{(\alpha, \beta)}(l+m, b ; c ; y z) d z \\
=\int_{0}^{1} z^{l-1}(1-z)^{m-1} \sum_{n=0}^{\infty}(l+m)_{n} \times \\
\frac{B_{p}^{(\alpha, \beta)}(b+n, c-b)}{B(b, c-b)} \frac{(y z)^{n}}{n !} d z .
\end{array}
$$

By change the order of integration and summation and using Beta integral, we get

$$
\begin{array}{r}
\int_{0}^{1} z^{l-1}(1-z)^{m-1} F_{p}^{(\alpha, \beta)}(l+m, b ; c ; y z) d z \\
=\sum_{n=0}^{\infty}(l+m)_{n} \frac{B_{p}^{(\alpha, \beta)}(b+n, c-b)}{B(b, c-b)} \frac{\Gamma(l+n) \Gamma(m)}{\Gamma(l+m+n)} \frac{(y)^{n}}{n !} \\
=\frac{\Gamma(l) \Gamma(m)}{\Gamma(l+m)} \sum_{n=0}^{\infty}(l)_{n} \frac{B_{p}^{(\alpha, \beta)}(b+n, c-b)}{B(b, c-b)} \frac{(y)^{n}}{n !}
\end{array}
$$

which, upon using (3), yields our desired result (6). This completes the proof of Theorem 1.

Theorem 2.If $\Re(s)>0, \Re(p) \geq 0$

and $\left|\frac{y}{s}\right|<1$ then:

$L\left\{z^{l-1} F_{p}^{(\alpha, \beta)}(a, b ; c ; y z)\right\}=\frac{\Gamma(l)}{s^{l}}{ }_{1} F_{p}^{(\alpha, \beta)}\left(a, l, b ; c ; \frac{y}{s}\right)$,

where the Laplace transform of $f(z)$ is defined as (see, [19]):

$L\{f(z)\}=\int_{0}^{\infty} e^{-s z} f(z) d z$,

provided that both sides of above result exist.

Proof. On using the definition (11) and applying (3), we get

$$
\begin{array}{r}
\int_{0}^{\infty} z^{l-1} e^{-s z} F_{p}^{(\alpha, \beta)}(a, b ; c ; y z) d z \\
=\int_{0}^{\infty} z^{l-1} e^{-s z} \sum_{n=0}^{\infty}(a)_{n} \frac{B_{p}^{(\alpha, \beta)}(b+n, c-b)}{B(b, c-b)} \frac{(y z)^{n}}{n !} d z .
\end{array}
$$

By change the of order of integration and summation and using Laplace transform, we get

$$
\begin{array}{r}
\int_{0}^{\infty} z^{l-1} e^{-s z} F_{p}^{(\alpha, \beta)}(a, b ; c ; y z) d z \\
=\sum_{n=0}^{\infty}(a)_{n} \frac{B_{p}^{(\alpha, \beta)}(b+n, c-b)}{B(b, c-b)} \frac{\Gamma(l+n)}{s^{l+n}} \frac{(y)^{n}}{n !},
\end{array}
$$

which, upon using (3), yields our desired result (10). 
Theorem 3.If $\rho, \delta \in \mathbb{C}, \Re(p) \geq 0$ and $\left|\frac{w}{\delta}\right|<1$, then the following Whittaker transform formula hold true:

$$
\begin{array}{r}
\int_{0}^{\infty} t^{\rho-1} e^{-\delta t / 2} W_{\lambda, \mu}(\delta t) F_{p}^{(\alpha, \beta)}(a, b ; c ; w t) d t \\
=\delta^{-\rho} \frac{\Gamma\left(\frac{1}{2}+\mu+\rho\right) \Gamma\left(\frac{1}{2}-\mu+\rho\right)}{\Gamma(1-\lambda+\rho)} \times \\
{ }_{2} F_{p, 1}^{(\alpha, \beta)}\left(a, \frac{1}{2}+\mu+\rho, \frac{1}{2}-\mu+\rho, b ; c, 1-\lambda+\rho ; \frac{w}{\delta}\right)(14)
\end{array}
$$

where, it being assumed that the member of the Whittaker transform exist.

Proof.Substituting $\delta t=v$ in LHS of (14), we get

$$
\begin{array}{r}
\int_{0}^{\infty}\left(\frac{v}{\delta}\right)^{\rho-1} e^{-v / 2} W_{\lambda, \mu}(v) \sum_{n=0}^{\infty}(a)_{n} \frac{B_{p}^{(\alpha, \beta)}(b+n, c-b)}{B(b, c-b)} \times \\
\frac{(w v)^{n}}{\delta^{n} \delta n !} d v .
\end{array}
$$

By change the of order of integration and summation, we get

$$
\begin{aligned}
= & \delta^{-\rho} \sum_{n=0}^{\infty}(a)_{n} \frac{B_{p}^{(\alpha, \beta)}(b+n, c-b)}{B(b, c-b)} \times \\
& \frac{w^{n}}{\delta^{n} n !} \int_{0}^{\infty} v^{\rho+n-1} e^{-v / 2} W_{\lambda, \mu}(v) d v .
\end{aligned}
$$

Now, we use the following integral formula involving the Whittaker function

$$
\begin{array}{r}
\int_{0}^{\infty} t^{v-1} e^{-t / 2} W_{\lambda, \mu}(t) d t \\
=\frac{\Gamma\left(\frac{1}{2}+\mu+v\right) \Gamma\left(\frac{1}{2}-\mu+v\right)}{\Gamma\left(\frac{1}{2}-\lambda+v\right)}, \\
\left(\Re(v \pm \mu)>-\frac{1}{2}\right)
\end{array}
$$

then equation (15) becomes in the following

$$
\begin{array}{r}
=\delta^{-\rho} \sum_{n=0}^{\infty}(a)_{n} \frac{B_{p}^{(\alpha, \beta)}(b+n, c-b)}{B(b, c-b)} \frac{w^{n}}{\delta^{n} n !} \times \\
\frac{\Gamma\left(\frac{1}{2}+\mu+\rho+n\right) \Gamma\left(\frac{1}{2}-\mu+\rho+n\right)}{\Gamma(1-\lambda+\rho+n)},
\end{array}
$$

which, upon using (3), yields our desired result (14). This completes the proof of Theorem 3.

\section{Fractional Calculus of the Generalized Gauss Hypergeometric Functions}

In view their importance and popularity in recent years, the theory of operators of fractional calculus has been developed widely and extensively (see, for example, [1-5, 9-12, 15-18; see also [20]). Here, in this section, we shall establish six fractional integral formulas for the generalized Gauss hypergeometric type functions $F_{p}^{(\alpha, \beta)}(a, b ; c ; z)$. The results are given in the form of the theorems and corollaries. The first two theorems are derived and then the remaining four results are deduced as their corollaries. For the purpose of these results, we use the following pair of Saigo hypergeometric operators of fractional integration.

For $x>0, \mu, v, \eta \in \mathbb{C}$ and $\Re(\mu)>0$, we have:

$$
\begin{gathered}
\left(I_{0, x}^{\mu, v, \eta} f(t)\right)(x)=\frac{x^{-\mu-v}}{\Gamma(\mu)} \int_{0}^{x}(x-t)^{\mu-1} \times \\
{ }_{2} F_{1}(\mu+v,-\eta ; \mu ; 1-t / x) f(t) d t, \\
\left(J_{x, \infty}^{\mu, v, \eta} f(t)\right)(x)=\frac{1}{\Gamma(\mu)} \int_{x}^{\infty}(t-x)^{\mu-1} t^{-\mu-v} \times \\
{ }_{2} F_{1}(\mu+v,-\eta ; \mu ; 1-x / t) f(t) d t,
\end{gathered}
$$

where, the ${ }_{2} F_{1}($.$) function occurring in the right-hand side$ of the above equations, is the special case of the well known generalized hypergeometric series ${ }_{p} F_{q}($.$) is given$ by (4).

The operator $I_{0, x}^{\mu, v, \eta}($.$) contains both the$ Riemann-Liouville and the Erdélyi-Kober fractional integral operators, by means of the following relationships:

$$
\begin{array}{r}
\left(R_{0, x}^{\mu} f(t)\right)(x)=\left(I_{0, x}^{\mu,-\mu, \eta} f(t)\right)(x) \\
=\frac{1}{\Gamma(\mu)} \int_{0}^{x}(x-t)^{\mu-1} f(t) d t
\end{array}
$$

and

$$
\begin{aligned}
& \left(E_{0, x}^{\mu, \eta} f(t)\right)(x)=\left(I_{0, x}^{\mu, 0, \eta} f(t)\right)(x) \\
& =\frac{x^{-\mu-\eta}}{\Gamma(\mu)} \int_{0}^{x}(x-t)^{\mu-1} t^{\eta} f(t) d t,
\end{aligned}
$$

where as the operator (19) unifies the Weyl type and the Erdélyi-Kober fractional integral operators. Indeed we have:

$$
\begin{array}{r}
\left(W_{x, \infty}^{\mu} f(t)\right)(x)=\left(J_{x, \infty}^{\mu,-\mu, \eta} f(t)\right)(x) \\
=\frac{1}{\Gamma(\mu)} \int_{x}^{\infty}(t-x)^{\mu-1} f(t) d t,
\end{array}
$$

and

$$
\begin{aligned}
& \left(K_{x, \infty}^{\mu, \eta} f(t)\right)(x)=\left(J_{x, \infty}^{\mu, 0, \eta} f(t)\right)(x) \\
= & \frac{x^{\eta}}{\Gamma(\mu)} \int_{x}^{\infty}(t-x)^{\mu-1} t^{-\mu-\eta} f(t) d t .
\end{aligned}
$$

In the sequel, we shall be using the following image formulas which are easy consequences of the operators 
(18) and (19) $([16,18])$ :

$$
\begin{array}{r}
\left(I_{0, x}^{\mu, v, \eta} t^{\lambda-1}\right)(x)=\frac{\Gamma(\lambda) \Gamma(\lambda-v+\eta)}{\Gamma(\lambda-v) \Gamma(\lambda+\mu+\eta)} \times \\
x^{\lambda-v-1} \quad(\lambda>0, \lambda-v+\eta>0),
\end{array}
$$

and

$$
\begin{aligned}
& \left(J_{x, \infty}^{\mu, v, \eta} t^{\lambda-1}\right)(x)=\frac{\Gamma(v-\lambda+1) \Gamma(\eta-\lambda+1)}{\Gamma(1-\lambda) \Gamma(v+\mu-\lambda+\eta+1)} \times \\
& x^{\lambda-v-1} \quad(\beta-\lambda+1>0, \eta-\lambda+1>0) \text {. }
\end{aligned}
$$

The Saigo fractional integrations of generalized Gauss hypergeometric type functions (3), are given by the following results:

Theorem 4.Let $\mu, v, \eta, \rho \in \mathbb{C}$ and $x>0$ be such that $\mathfrak{R}(\mu)>0, \mathfrak{R}(p) \geq 0, \mathfrak{R}(\rho)>0$ and $\mathfrak{R}(\rho)>$ $\max [0, \mathfrak{R}(v-\eta)]$, then:

$$
\begin{aligned}
& \left(I_{0, x}^{\mu, v, \eta}\left[t^{\rho-1} F_{p}^{(\alpha, \beta)}(a, b ; c ; e t)\right]\right)(x) \\
& =x^{\rho-v-1} \frac{\Gamma(\rho) \Gamma(\rho-v+\eta)}{\Gamma(\rho+\mu+\eta) \Gamma(\rho-v)} \times \\
& { }_{2} F_{p, 2}^{(\alpha, \beta)}\left[\begin{array}{ll}
a, b, \rho, \rho-v+\eta ; & e x \\
c, \rho-v, \rho+\mu+\eta ;
\end{array}\right] .
\end{aligned}
$$

Proof.For convenience sake, we denote the left-hand side of the result (26) by $\mathscr{I}$. Using definition (3), and then changing the order of integration and summation, which is valid under the condition of Theorem 4, we find that

$$
\mathscr{I}=\left(I_{0, x}^{\mu, v, \eta}\left[t^{\rho-1} \sum_{n=0}^{\infty} a_{n} \frac{B_{p}^{(\alpha, \beta)}(b+n, c-b)}{B(b, c-b)} \frac{(e t)^{n}}{n !}\right]\right)(x)
$$$$
=\sum_{n=0}^{\infty} a_{n} \frac{B_{p}^{(\alpha, \beta)}(b+n, c-b)}{B(b, c-b)} \frac{e^{n}}{n !}\left(I_{0, t}^{\mu, v, \eta}\left\{t^{\rho+n-1}\right\}\right)(x) .
$$

Now, on making use of result (24), we obtain

$$
\begin{gathered}
\mathscr{I}=x^{\rho-v-1} \sum_{n=0}^{\infty} a_{n} \frac{B_{p}^{(\alpha, \beta)}(b+n, c-b)}{B(b, c-b)} \times \\
\frac{\Gamma(\rho+n) \Gamma(\rho-v+\eta+n)}{\Gamma(\rho-v+n) \Gamma(\rho+\mu+\eta+n)} \frac{(e x)^{n}}{n !}
\end{gathered}
$$

This, in accordance with definition (3), gives the required result (26).
Theorem 5.Let $\mu, v, \eta, \rho \in \mathbb{C}, x>0$ and satisfying the inequalities $\mathfrak{R}(\mu)>0, \mathfrak{R}(p) \geq 0, \mathfrak{R}(\rho)>0, \mathfrak{R}(\rho)<$ $1+\min \{\Re(\eta), \Re(v)\}$, then:

$$
\begin{array}{r}
\left(J_{x, \infty}^{\mu, v, \eta}\left[t^{\rho-1} F_{p}^{(\alpha, \beta)}\left(a, b ; c ; \frac{e}{t}\right)\right]\right)(x) \\
=x^{\rho-v-1} \frac{\Gamma(1-\rho+v) \Gamma(1-\rho+\eta)}{\Gamma(1-\rho) \Gamma(1-\rho-\eta+v+\mu)} \times \\
{ }_{2} F_{p, 2}^{(\alpha, \beta)}\left[\begin{array}{c}
a, b, 1-\rho+v, 1-\rho+\eta ; e \\
c, 1-\rho, 1-\rho+\mu+v-\eta ; x
\end{array}\right] .
\end{array}
$$

Proof.Proceeding as in Theorem 4, and taking operator (19) and result (25) into account, one can easily prove the above theorem. Therefore, we omit the details of the proof of this theorem.

Interestingly, on setting $v=0$ and employing the relations (21) and (23), the Theorems 4 . and 5. yields to the following corollaries.

Corollary 1.Let $\mu, \eta, \rho \in \mathbb{C}$ and $x>0$ be such that $\mathfrak{R}(\mu)>0, \mathfrak{R}(p) \geq 0, \mathfrak{R}(\rho)>0$ and $\mathfrak{R}(\rho)>\mathfrak{R}(-\eta)$, then the rigt-side Erdélyi-Kober fractional integrals of the generalized Gauss hypergeometric type functions are given by:

$$
\begin{array}{r}
\left(E_{0, x}^{\mu, \eta}\left[t^{\rho-1} F_{p}^{(\alpha, \beta)}(a, b ; c ; e t)\right]\right)(x) \\
=x^{\rho-1} \frac{\Gamma(\rho-\eta)}{\Gamma(\rho+\mu+\eta)}{ }_{1} F_{p, 1}^{(\alpha, \beta)}\left[\begin{array}{c}
a, b, \rho-\eta ; \\
c, \rho+\mu+\eta ;
\end{array}\right] .
\end{array}
$$

Corollary 2.Let $\mu, \eta, \rho \in \mathbb{C}, x>0$ and satisfying the inequalities

$\mathfrak{R}(\mu)>0, \Re(p) \geq 0, \mathfrak{R}(\rho)>0, \mathfrak{R}(\rho)<1+\mathfrak{R}(\eta)$, then:

$$
\begin{array}{r}
\left(K_{x, \infty}^{\mu, \eta}\left[t^{\rho-1} F_{p}^{(\alpha, \beta)}\left(a, b ; c ; \frac{e}{t}\right)\right]\right)(x) \\
=x^{\rho-1} \frac{\Gamma(1-\rho+\eta)}{\Gamma(1-\rho-\eta+\mu)}{ }_{1} F_{p, 1}^{(\alpha, \beta)}\left[\begin{array}{c}
a, b, 1-\rho+\eta ; e \\
c, 1-\rho+\mu-\eta ; x
\end{array}\right] .
\end{array}
$$

Further, if we replace $v$ by $-\mu$ and make use of the relations (20) and (22), in the Theorems 4. and 5., we obtain yet another corollaries providing Riemann-Liouville and Weyl fractional integrals of the generalized Gauss hypergeometric type function $F_{p}^{(\alpha, \beta)}$, as follows:

Corollary 3.Let $\mu, \rho \in \mathbb{C}$ and $x>0$, such that $\Re(\mu)>$ $0, \mathfrak{R}(p) \geq 0, \mathfrak{R}(\rho)>0$, then:

$$
\begin{array}{r}
\left(R_{0, x}^{\mu}\left[t^{\rho-1} F_{p}^{(\alpha, \beta)}(a, b ; c ; e t)\right]\right)(x) \\
=x^{\rho+\mu-1} \frac{\Gamma(\rho)}{\Gamma(\rho+\mu)}{ }_{1} F_{p, 1}^{(\alpha, \beta)}\left[\begin{array}{c}
a, b, \rho ; \\
c, \rho+\mu, ;
\end{array}\right] .
\end{array}
$$


Corollary 4.Let $\mu, \rho \in \mathbb{C}, x>0$ and satisfying the inequalities $\Re(\mu)>0, \Re(p) \geq 0, \Re(\rho)>0$, then:

$$
\begin{array}{r}
\left(W_{x, \infty}^{\mu}\left[t^{\rho-1} F_{p}^{(\alpha, \beta)}\left(a, b ; c ; \frac{e}{t}\right)\right]\right)(x) \\
=x^{\rho+\mu-1} \frac{\Gamma(1-\rho-\mu)}{\Gamma(1-\rho)}{ }_{1} F_{p, 1}^{(\alpha, \beta)}\left[\begin{array}{r}
a, b, 1-\rho-\mu ; e \\
c, 1-\rho, ;
\end{array}\right] .
\end{array}
$$

\section{Concluding Remarks}

In this section, we consider some consequences of the main results derived in the preceding sections. If we set $\alpha=\beta$ in (26) and (29) respectively, then by the known formula due to Chadudhary et al. (see, e.g., [8]), the Theorems 4. and 5. yields to the following corollaries:

Corollary 5.Let $\mu, v, \eta, \rho \in \mathbb{C}$ and $x>0$ be such that $\Re(\mu)>0, \Re(p) \geq 0, \Re(\rho)>0$ and $\Re(\rho)>$ $\max [0, \mathfrak{R}(v-\eta)]$, then:

$$
\begin{aligned}
& \left(I_{0, x}^{\mu, v, \eta}\left[t^{\rho-1} F_{p}(a, b ; c ; e t)\right]\right)(x) \\
= & x^{\rho-v-1} \frac{\Gamma(\rho) \Gamma(\rho-v+\eta)}{\Gamma(\rho+\mu+\eta) \Gamma(\rho-v)} \\
& { }_{2} F_{p, 2}\left[\begin{array}{c}
a, b, \rho, \rho-v+\eta ; \\
c, \rho-v, \rho+\mu+\eta ;
\end{array}\right] .
\end{aligned}
$$

Corollary 6.Let $\mu, v, \eta, \rho \in \mathbb{C}, x>0$ and satisfying the inequalities $\Re(\mu)>0, \Re(p) \geq 0, \Re(\rho)>0, \Re(\rho)<1+$ $\min \{\mathfrak{R}(\eta), \mathfrak{R}(v)\}$, then:

$$
\begin{array}{r}
\left(J_{x, \infty}^{\mu, v, \eta}\left[t^{\rho-1} F_{p}\left(a, b ; c ; \frac{e}{t}\right)\right]\right)(x) \\
=x^{\rho-\beta-1} \frac{\Gamma(1-\rho+v) \Gamma(1-\rho+\eta)}{\Gamma(1-\rho) \Gamma(1-\rho-\eta+v+\mu)} \\
{ }_{2} F_{p, 2}\left[\begin{array}{c}
a, b, 1-\rho+v, 1-\rho+\eta ; e \\
c, 1-\rho, 1-\rho+\mu+v-\eta ; x
\end{array}\right],
\end{array}
$$

which are also believe to be new.

Furthermore, if we set $p=0$ then, or make use of result (3), Theorems 1 to 5 yield the various integral transforms and fractional integral formulas for the generalized hypergeometric function ${ }_{2} F_{1}$.

The generalized Gauss hypergeometric type functions defined by (3), possess the advantage that most of the known and widely-investigated special functions are expressible also in terms of the generalized Gauss hypergeometric functions $F_{p}^{(\alpha, \beta)}$ (for some interesting examples and applications, see [13, Chapter 3 and 5 ]). Therefore, we conclude this paper with the remark that, the results deduced above are significant and can lead to yield numerous other integral transforms and fractional integral formulas involving various special functions by the suitable specializations of arbitrary parameters in the theorems. More importantly, they are expected to find some applications in probability theory and to the solutions of fractional differential and integral equations. The results thus derived in this paper are general in character and likely to find certain applications in the theory of special functions.

\section{References}

[1] P. Agarwal, Fractional integration of the product of two multivariables $H$-function and a general class of polynomials, In: Adva. Appl. Math. Approx. Theo., (2011) (Springer Proc. in Mathematics and Statistics), 41, 359-374 (2013).

[2] P. Agarwal, Further results on fractional calculus of Saigo operators, Appl. Appl. Math., 7, 585-594 (2012).

[3] P. Agarwal, Generalized fractional integration of the $\bar{H}$ function, Le Matematiche, LXVII, 107-118 (2012).

[4] P. Agarwal and S. Jain, Further results on fractional calculus of Srivastava polynomials, Bull. Math. Anal. Appl., 3, 167174 (2011).

[5] P. Agarwal and S.D. Purohit, The unified pathway fractional integral formulae, J. Fract. Calc. Appl., 4, 1-8 (2013).

[6] P. Agarwal, M. Chand and S.D. Purohit, A note on generating functions involving generalized Gauss hypergeometric functions, Nat. Acad. Sci. Lett., (In press).

[7] M.A. Chaudhry, A. Qadir, M. Rafique and S.M. Zubair, Extension of EulerŁ beta function, J. Comput. Appl. Math., 78, 19-32 (1997).

[8] M.A.Chaudhry, A. Qadir, H.M. Srivastava and R.B. Paris, Extended hypergeometric and confluent hypergeometric functions, Appl. Math. and Comput., 159, 589-602 (2004).

[9] A.A. Kilbas, Fractional calculus of the generalized Wright function, Fract. Calc. Appl. Anal., 8, 113-126 (2005).

[10] A.A. Kilbas, H.M. Srivastava, J.J. Trujillo, Theory and Applications of Fractional Differential Equations, NorthHolland Mathematical Studies, vol. 204, Elsevier (NorthHolland) Science Publishers, Amsterdam, London and New York, (2006).

[11] V. Kiryakova, On two Saigo's fractional integral operators in the class of univalent functions, Fract. Calc. Appl. Anal., 9, 160-176 (2006).

[12] V. Kiryakova, A brief story about the operators of the generalized fractional calculus, Fract. Calc. Appl. Anal., 11, 203-220 (2008).

[13] E.Özergin, Some properties of hypergeometric functions,Ph.D. Thesis, Eastern Mediterranean University, North Cyprus, February (2011).

[14] E. Özergin, M. A. Özarslan and A. Altin, Extension of gamma, beta and hypergeometric functions, J. Comput. Appl. Math., 235, 4601-4610 (2011).

[15] M.Saigo, On generalized fractional calculus operators, In: Recent Advances in Applied Mathematics (Proc. Internat. Workshop held at Kuwait Univ.) Kuwait Univ., Kuwait, 441450 (1996).

[16] M. Saigo, A remark on integral operators involving the Gauss hypergeometric functions, Math. Rep. Kyushu Univ., 11, 135-143 (1978).

[17] M. Saigo, A certain boundary value problem for the EulerDarboux equation I, Math. Japonica, 24, 377-385 (1979). 
[18] M. Saigo and N. Maeda, More generalization of fractional calculus, In: Transform Metods and Special Functions, Varna, 1996 (Proc. 2nd Intern. Workshop, Eds. P. Rusev, I. Dimovski, V. Kiryakova), IMI-BAS, Sofia, 386-400 (1998).

[19] I. N. Sneddon, The Use of Integral Transforms, Tata McGraw-Hill, New Delhi, (1979).

[20] H. M. Srivastava and P.Agarwal, Certain fractional integral operators and the generalized incomplete Hypergeometric functions, Appl. Appl. Math. (In press).

[21] H. M. Srivastava and J. Choi, Zeta and q-Zeta Functions and Associated Series and Integrals, Elsevier Science Publishers, Amsterdam, London and New York, (2012).

[22] H.M. Srivastava and P.W. Karlsson, Multiple Gaussian Hypergeometric Series, Ellis Horwood Limited, (1985).

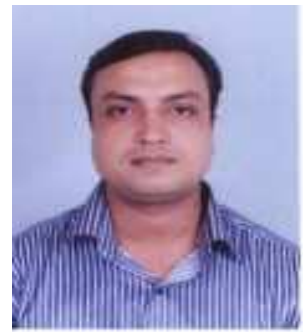

Praveen Agarwal

obtained his M.Sc., M. Phil and Ph.D. (work carried out from MNIT, Jaipur) degrees from the Faculty of Science at Rajasthan University, Jaipur, India. He also had a Research Fellowship of MNIT, Jaipur and then worked in the Department of Mathematics, Global Institute of Technology, Jaipur, India. Currently, he is Associate Professor and Head at the Department of Mathematics, Anand International College of Engineering, Jaipur, India. His research interest includes special functions, fractional calculus, integral transforms, basic hypergeometric series and mathematical physics. $\mathrm{He}$ has published more than 55 research papers in international esteemed journals. $\mathrm{He}$ is Reviewer for Mathematical Reviews, USA (American Mathematical Society), and Zentralblatt MATH, Berlin, since last two years. He is member of editorial board for number of international mathematical and interdisciplinary journals. 